Artículo de revisión

\title{
Prevalencia y periodicidad del virus sincitial respiratorio en Colombia
}

\author{
Juliana Barbosa ${ }^{1}$, Beatriz Parra², Liliana Alarcón ${ }^{3}$, Flor Inés Quiñones ${ }^{4}$, Eduardo López ${ }^{2,5}$, Manuel Antonio Franco ${ }^{6, * *}$ \\ ${ }^{1}$ Instituto Nacional de Salud, Bogotá, D.C., Colombia \\ ${ }^{2}$ Universidad del Valle, Cali, Colombia \\ ${ }^{3}$ Programa Ampliado de Inmunizaciones de la Secretaría de Salud de Cali, Colombia \\ ${ }^{4}$ Secretaría de Salud Pública de Cali, Colombia \\ ${ }^{5}$ Centro de Estudios en Infectología Pediátrica, Cali, Colombiaisêpi \\ ${ }^{6}$ Instituto de Genética Humana, Facultad de Medicina, Pontificia Universidad Javeriana, Bogotá, Colombia
}

\begin{abstract}
Resumen
El virus sincitial respiratorio es uno de los principales agentes causantes de hospitalizaciones en niños menores de 5 años en todo el mundo y es responsable de una significativa mortalidad en los países en vías de desarrollo. Los resultados de estudios recientes sobre la inmunidad antiviral han generado un ambiente de optimismo sobre la posibilidad de lograr una vacuna antiviral segura y eficaz en un futuro cercano. En este contexto, uno de los objetivos del seminario taller sobre integración intersectorial de la investigación en salud, organizado por el Cideim, fue detectar las necesidades de investigación para facilitar la introducción de las vacunas contra el virus sincitial respiratorio en Colombia y, en particular, hacer un análisis de la carga de la enfermedad producida por este agente en Colombia. Como contribución a este último objetivo, en el presente trabajo se hizo la revisión de 36 estudios epidemiológicos y clínicos sobre el virus sincitial respiratorio realizados en el país en los últimos 40 años, con el fin de evaluar históricamente la prevalencia de la enfermedad por este patógeno y la periodicidad reportada en las diferentes ciudades de Colombia. Aunque en varias ciudades existe un pico en la presentación del virus entre marzo y abril, particularmente en Bogotá, la ciudad del país con el mayor número de estudios, en otras ciudades, como Cali, este no parece ser el caso. A pesar de que existe heterogeneidad en la prevalencia de las infecciones por el virus sincitial respiratorio (rango de 7 a $41 \%$ ) dependiendo, sobre todo, del tipo de estudio, en los últimos 40 años este ha sido el principal agente responsable de la infección respiratoria baja en niños menores de 5 años en Colombia. (C) 2017. Acad. Colomb. Cienc. Ex. Fis. Nat.
\end{abstract}

Palabras claves: Virus sincitial respiratorio; Periodicidad; Prevalencia; Colombia; Infecciones respiratorias; Niño.

Prevalence and periodicity of respiratory syncytial virus in Colombia

\begin{abstract}
Respiratory syncytial virus is one of the main agents responsible for hospitalizations in children under 5 years of age worldwide and is responsible for significant mortality in developing countries. Recent studies on antiviral immunity have created an atmosphere of optismism about the possibility of achieving a safe and effective antiviral vaccine in the near future. In this context, during the course of the Seminar/Workshop: "Intersectoral integration in health research" organized by CIDEIM, one of the objectives was to identify research needs to facilitate the introduction of respiratory syncytial virus vaccines in Colombia and particularly to estimate the disease burden by this agent in the country. In this context, the present work revised the information reported by epidemiologial and clinical studies on respiratory syncytial virus conducted in the country within the past 40 years to describe the disease prevalence and the temporal distribution of this respiratory pathogen in different cities of Colombia. Although in several cities there is a peak in the presentation of the virus between March and April, especially in Bogotá, the city with the greatest number of studies, this does not seem to be the case in other cities, for example, Cali. Despite the fact that there is heterogeneity in the prevalence of infection by respiratory sincitial virus (range: $7 \%-41 \%$ ) depending on the type of study, for the last 40 years the virus has been the main agent responsible for lower respiratory tract infections in children under 5 years in Colombia. (C) 2017. Acad. Colomb. Cienc. Ex. Fis. Nat.
\end{abstract}

Key words: Respiratory Syncytial Virus; Periodicity; Prevalence; Colombia; Respiratory infections; Children.

\footnotetext{
*Correspondencia:

Manuel Antonio Franco Cortés, mafranco@javeriana.edu.co; Recibido: 09 de agosto de 2017; Aceptado: 03 de noviembre de 2017
} 
El virus sincitial respiratorio (VSR) es uno de los principales agentes causantes de hospitalizaciones en niños menores de 5 años en todo el mundo y es responsable de tasas significativas de mortalidad en los países en vías de desarrollo (Graham, 2016). El desarrollo de una vacuna contra este agente se ha visto afectado por el hecho de que los niños a quienes se les administró la primera vacuna en los años 60 presentaron una infección más grave que los no vacunados (Higgins, et al., 2016; Modjarrad, et al., 2015). Sin embargo, los estudios recientes sobre la inmunidad antiviral han generado un ambiente de optimismo sobre la posibilidad de lograr una vacuna contra el VSR segura y eficaz en un futuro cercano (Graham, 2016). Además, dado que la inmunidad materna puede proteger a los neonatos (Chu, et al., 2014), que constituyen una de las poblaciones más vulnerables, se ha planeado administrar la vacuna a las madres durante el embarazo y disminuir indirectamente el riesgo de enfermedad grave por VSR de los niños durante sus primeros meses de vida. Se espera, entonces, que las primeras vacunas que se autoricen se apliquern en mujeres embarazadas y en personas de la tercera edad, otra población particularmente sensible al virus (Giersing, et al., 2016). En este sentido, hoy están en desarrollo 60 vacunas dirigidas precisamente a estos dos grupos de población (Giersing, et al., 2016; Graham, 2016; Higgins, et al., 2016), y aunque la mayoría están en una fase preclínica, 16 de ellas ya están en la fase clínica. Novavax tiene ocho vacunas entre las fases uno y tres, Medimmune tiene cuatro entre las fases uno y dos b y GSK, cuatro en las fases uno y dos (Giersing, $\boldsymbol{e t}$ al., 2016; Higgins, et al., 2016). Se espera que las primeras vacunas se aprueben aproximadamente dentro de cuatro años (Giersing, et al., 2016; Higgins, et al., 2016).

En este contexto, durante el seminario-taller sobre "Integración intersectorial en la investigación para la salud" organizado por el CIDEIM del 23 al 24 de junio de 2016 en Cali, uno de los objetivos fue establecer las necesidades de investigación para facilitar la introducción de las vacunas contra el VSR en Colombia. El primer aspecto que se discutió fue la necesidad de hacer un análisis de la morbilidad y la mortalidad en casosde infección respiratoria producida por el VSR en Colombia, pues esto permitiría tener una estimación histórica de la carga de enfermedad por el VSR con una caracterización de su periodicidad a nivel nacional, así como determinar los predictores de su gravedad. El presente trabajo pretende contribuir a este objetivo mediante la revisión de los estudios sobre el VSR realizados en el país, con el fin de estimar la prevalencia de la enfermedad por este patógeno y la periodicidad reportada en las ciudades del país. Se consultaron las bases de datos Medline, Embase, SciELO y Google Scholar, así como a expertos. Solo se tuvieron en cuenta los documentos con acceso a su texto completo. Aunque nuestro propósito era documentar todos los trabajos epidemiológicos y clínicos sobre VSR en el país, somos conscientes de que podría haber otros trabajos que no se detectaron.
La información se organizó y se presenta en dos apartes: 1) los trabajos gubernamentales sobre el tema, con especial énfasis en la vigilancia de VSR que realiza el Instituto Nacional de Salud (INS) , y 2) los estudios hechos en el país agrupados según la ciudad en que fueron publicados.

\section{Esfuerzos gubernamentales para el seguimiento y el manejo del VSR en Colombia}

Desde 1997 el INS lleva a cabo la vigilancia centinela de los virus respiratorios causantes de la infección respiratoria aguda (IRA) (Barbosa, et al., 2014), la cual se ajusta a los protocolos definidos, el primero de ellos de 2010, modificado en el 2013 y actualizado por última vez en el 2016.

Las estrategias empleadas en la vigilancia son las siguientes: 1) vigilancia centinela de enfermedades similares a la influenza (ESI) y de la infección respiratoria aguda grave (IRAG); 2) vigilancia de la morbilidad por IRA hospitalaria y ambulatoria; 3) vigilancia de IRAG inusitada, y 4) vigilancia de la mortalidad por IRA en menores de cinco años.

Uno de los objetivos de la primera de estas estrategias es "Determinar la carga de la infección respiratoria aguda en la prestación de servicios de salud en donde se efectúa vigilancia centinela de ESI - IRAG”. A partir del 2006 el INS descentralizó el diagnóstico de los virus respiratorios mediante inmunofluorescencia y lo transfirió a 12 laboratorios de salud pública del país con 40 sitios centinelas (Barbosa, et al., 2014). A partir del 2013 se hace en el INS y en otros laboratorios de apoyo el diagnóstico de varios virus respiratorios (incluidoel VSR) mediante PCR con transcriptasa inversa (RT-PCR) en tiempo real (Barbosa, et al., 2014), Y desde el 2007, (año en que se comenzó la vigilancia de ESI, el VSR ha sido el principal agente detectado en menores de 5 años con diagnóstico de ESI (Barbosa, et al., 2014). Los datos de esta vigilancia se publican en los boletines epidemiológicos del INS (http:// www.ins.gov.co) y, además, en informes anuales en los que se consolidan los datos de todos los eventos de interés en salud pública, incluidas las enfermedades respiratorias. En ellos se ha reportado anualmente (desde el 2009 hasta el 2016) la circulación del VSR en Colombia. Para los años 2009, 2010, 2011, 2012, 2013, 2014, 2015 y 2016 se detectó el VSR en 45, 50, 45,3, 59, 36, 38,9, 51,9 y 41,0\% de las muestras, respectivamente. Además, estos resultados se reportan a la Organización Panamericana de la Salud (OPS), que los tiene disponibles en su sitio de Internet (http://ais. paho.org/phip/viz/ed_flu.asp; fecha de consulta: 30 octubre de 2016). Es de anotar que los datos de Colombia en el sitio de la OPS provienen del INS, por lo que solo representan un subgrupo de los del Sivigila. Con los datos del INS se han publicado documentos sobre el tema (Barbosa, et al., 2014) y un artículo exhaustivo (14.870 muestras de pacientes con IRA) sobre la vigilancia del VSR en toda Colombia del 2000 al 2011 (Barbosa-Ramírez, et al., 2014). El subtipo A del VSR fue dominante con intervalos de dos años y el subtipo B con intervalos de un año. El 85,3 \% de las infecciones 
con VSR se presentó en niños menores de 5 años. A nivel nacional los picos de prevalencia del VSR coincidieron con el primer periodo de lluvia del país (abril-mayo) y un segundo pico más leve, entre octubre y noviembre (Barbosa, et al., 2014). Otro de los esfuerzos gubernamentales encaminados a combatir el VSR y que permiten dar una idea de los costos que este origina, son los estudios sobre el palivizumab, anticuerpo monoclonal neutralizante que ha demostrado tener un efecto antiviral (Higgins, et al., 2016), pero que es muy costoso (COP $\$ 18.343 .493,126$ al año para el 2008). Por ello el Ministerio de Salud contrató un estudio de costoefectividad de la profilaxis con palivizumab contra el VSR en niños prematuros a cargo de un equipo de la Pontificia Universidad Javeriana (Rueda, et al., 2013). Se determinó que el uso rutinario del anticuerpo no era una medida costoefectiva en estos niños debido a su alto costo y la poca eficacia comprobada para reducir la mortalidad o para la prevención primaria de la infección. Una de las limitaciones de este estudio fue que las estimaciones de la carga enfermedad se basaron en un estudio que no se había hecho en Colombia (Rueda, et al., 2013), lo cual confirma la necesidad de estimar este indicador con los datos del país. Utilizando en parte los resultados de este estudio, el Ministerio de Salud emitió un documento con recomendaciones sobre el uso del palivizumab (Ministerio de Salud, 2014) en prematuros con mayor riesgo de enfermedad grave por VSR. En dicho documento se incluye un algoritmo para orientar la administración de este medicamento en niños menores de 32 semanas de edad de gestación, o en los menores de 35 semanas con displasia broncopulmonar o que dependan de la administración de oxígeno.

\section{Estudios sobre la prevalencia y la incidencia del VSR en Colombia}

La epidemiología y las características clínicas de la infección por VSR en Colombia son similares a las de otros países en vías de desarrollo de America Latina y el mundo (Bardach, et al., 2014; Weber, et al., 1998). Sin embargo, en todos los países de América con estaciones, los picos epidémicos del VSR son bien marcados y se asociados con el invierno, en tanto que en los países de la América tropical la infección ocurre a lo largo de todo el año con algunos picos en épocas de lluvia (Bardach, et al., 2014; Weber, et al., 1998). Esto puede comprobarse con los datos que reporta la OPS sobre infección por VSR en las Américas, en los cuales se observan diferencias notables en la presentación epidemiológica entre los países del Caribe, los de Centroamérica, los de la región Andina y los del Cono Sur (http://ais.paho.org/phip/viz/ed_ flu.asp; fecha de consulta: 30 de octubre 2016).

En Colombia se vienen publicando estudios que evalúan la presencia del VSR en el país desde las décadas de 1970 y 1980 (Berman, et al., 1983; Escobar, et al., 1976; Núñez, et al., 1988). Con excepción del artículo del INS ya mencionado, en el que se reportan los resultados de la vigilancia del VSR a nivel nacional, la gran mayoría de estudios registra los resultados de ciudades específicas, y no hay estudios regionales o multicéntricos. La excepción es un estudio con un número relativamente escaso de participantes (717 en total y entre 47 y 296 por cada una de las seis ciudades incluidas: Barranquilla, Bogotá, Bucaramanga, Cali, Medellín y Pereira), en el cual se evaluaron los agentes etiológicos de la infección respiratoria baja en niños hospitalizados menores de un año. El VSR fue responsable de una de cada tres hospitalizaciones por infección respiratoria baja, con una periodicidad variable según la ciudad difícil de precisar por el escaso número de pacientes en algunas de ellas (Piñeros, et al., 2013).

\section{Estudios en Cali}

En el primer estudio que pudimos documentar sobre la prevalencia del VSR en Colombia (realizado en 1976 en Cali), se encontró una baja prevalencia del virus, probablemente debido a la metodología usada (seroconversión de anticuerpos mediante fijación de complemento) (Escobar, et al., 1976). Posteriormente, se reportaron dos estudios que estimaron la incidencia de la infección respiratoria aguda en servicios de atención ambulatoria, principalmente en niños menores de 5 años pertenecientes a estratos socioeconómicos bajos, en los cuales se identificaron los agentes etiológicos más frecuentemente asociados y se estableció la periodicidad. En el estudio titulado "Enfermedad respiratoria aguda del tracto respiratorio inferior", se analizaron 1.229 pacientes durante 25 meses entre el 1 febrero de 1977 y el 28 febrero de 1979 y se diagnosticó la infección viral en $20 \%$ de ellos. El VSR se asoció a $9 \%$ de los casos y fue el patógeno respiratorio más frecuentemente identificado. La mayoría de las infecciones por VSR se diagnosticó en niños menores de 2 años de edad con bronquiolitis o neumonía, y más de $40 \%$ de los casos de infección con VSR ocurrió en infantes antes del primer año de vida (Berman, et al., 1983). El promedio de días de enfermedad por VSR fue de 18 días y en los casos de IRA baja (bronquiolitis y neumonías), la mayor morbilidad se asoció con desnutrición grave. En el estudio se evaluaron las tasas de infección del virus de la influenza, la parainfluenza, el adenovirus y el VSR y su relación con los periodos de lluvia y los meses del año. La circulación del VSR fue mayor durante los meses de diciembre, enero y febrero en los dos años del estudio y no siempre coincidió con los períodos de más lluvia en Cali. El adenovirus fue el segundo virus más frecuente y circuló durante todo el año.

En otro estudio prospectivo hecho entre 1986 y 1988, se evaluaron los episodios de IRA en una cohorte de 340 niños desde el nacimiento hasta la edad de 17 meses (Borrero, et al., 1990). La incidencia de episodios de IRA por niño y año fue de 6,6, lo que sugería la presencia de reinfecciones múltiples. El VSR fue el agente viral más frecuentemente aislado de los cultivos de aspirados nasofaríngeos en niños con episodios de IRA baja, y se lo detectó casi tres veces más que los otros virus. El VSR constituyó el principal agente viral en los niños durante sus primeros 17 meses de 
vida. Un hallazgo interesante para el diagnóstico diferencial de IRA viral en niños fue que las retracciones y la frecuencia respiratoria de más de 50 por minuto fueron signos clínicos distintivos de los casos de IRA baja.

Un reciente estudio internacional sobre VSR, que en Colombia se hizo para la ciudad de Cali, merece especial mención, pues permite comparar la incidencia y la prevalencia del virus en Colombia con las de otros países del mundo. En el marco de un ensayo de una vacuna contra la influenza pandémica, se estudiaron niños sanos de 6 meses a $<10$ años de edad en el momento de la vacunación en Australia, Brasil, Colombia, Costa Rica, México, Filipinas, Singapur y Tailandia entre febrero del 2010 y agosto del 2011. En el estudio se hizo vigilancia activa prospectiva del VSR en niños con episodios de ESI hospitalizados o no, lo cual permitió evaluar la incidencia y la prevalencia de la enfermedad leve y grave (Nolan, et al., 2015). La prevalencia promedio del VSR en casos de ESI en Colombia (Cali) fue similar a la de otros países (9,7 \%). En Colombia, el 0, 4,6, 8,6,10,6, 28,3 y $47,9 \%$ de los participantes con ESI de $<6,6$ a11, 12 a 23, 24 a 35,36 a 59 y $>60$ meses de edad, respectivamente, tenían el VSR. La incidencia para Colombia fue relativamente baja y similar a la de Costa Rica, México y Tailandia (13 a 15 por 100 años por persona). La periodicidad varió según el país y en Colombia no fue clara.

En resumen, el VSR fue el principal agente etiológico causante de bronquiolitis y neumonías en menores de 2 años en Cali; otro hallazgo común de los estudios fue un mayor riesgo de IRA en varones que en niñas, lo cual no se estimó para el VSR, pero coincide con lo reportado a nivel mundial para este virus.

\section{Estudios en Medellín y Manizales}

Uno de los primeros estudios que se realizaron en el país usando pruebas rápidas de inmunofluorescencia para identificar virus respiratorios se hizo en Manizales, Caldas, entre el 1 de octubre de 1985 y el 22 de julio de 1986 (Núñez, et al., 1988). Se evalúo la etiología viral en secreciones traqueales de 60 niños de 1 a 60 meses de edad hospitalizados con IRA baja. En $33 \%(20 / 60)$ de ellos se identificaron virus, y en $3,3 \%$, Chlamydia trachomatis (2/60). El principal agente etiológico de IRA baja fue el VSR, identificado en $25 \%$ $(15 / 60)$ de los niños menores de 5 años. Sin embargo, en $42 \%$ del total se encontraron bacterias como único agente etiológico. Es importante resaltar que $50 \%$ de las IRA virales correspondieron a coinfecciones con bacterias.

En un estudio descriptivo realizado en Medellín se evaluó la frecuencia de VSR en niños hospitalizados con IRA baja entre abril de 1994 y abril de 1995. De los 103 pacientes menores de un año incluidos en el estudio, 43 $(41,7 \%)$ tenían infección por VSR. El mayor número de ingresos tanto por IRA como por la infección por VSR se dio en los meses lluviosos, y el pico de incidencia fue entre noviembre, diciembre y enero (Bedoya, et al., 1996).
En un estudio descriptivo realizado en el Hospital Pablo Tobón Uribe de de Medellín entre el 2007 y el 2012, se evaluó la frecuencia de aislamiento de virus respiratorios en 2.039 niños (1.364 eran menores de 5 años) con enfermedad similar a la influenza, de los cuales solo el $16 \%$ requirió hospitalización. Del total de participantes, 737 (36\%) fueron positivos para virus respiratorios. Es de anotar que el diagnóstico de influenza se hizo mediante aislamiento en cultivo celular y RT-PCR, mientras que los otros virus solo se identificaron en cultivo celular. En la mayoría de los casos en los que se identificó un virus, este fue el de influenza (57,8 \%) seguido del adenovirus (19,1\%), el de parainfluenza $(14,9 \%)$ y tan solo en $18(2,4 \%)$ casos se aisló el VSR (Arango, et al., 2015). La baja positividad para el VSR, comparada con el estudio anterior en Medellín y lo esperado en este grupo de edad, se atribuyó al uso de una línea celular Madin-Darby canine kidney (MDCK), en la que crece particularmente bien el virus de la influenza pero no el VSR, así como al hecho de que las muestras fueron congeladas para su posterior procesamiento en Perú.

\section{Estudios en Bogotá}

La Fundación Santafé realizó en Bogotá un estudio retrospectivo piloto en el cual se analizaron 3.193 consultas en el servicio de urgencias de su clínica entre 1990 y 1994. Se encontró que las afecciones respiratorias más comunes aparecían entre abril y mayo y los autores concluyeron que este patrón no coincidía con la circulación viral en el hemisferio norte ni enel hemisferio sur, como tampoco con lo descrito en otras zonas ecuatoriales del mundo. De 94 pacientes diagnosticados con bronquiolitis entre diciembre de 1993 y julio de 1994, se tomaron pruebas para VSR en 46; en el primer semestre fue positivo el $79 \%(27 / 34)$ y en el segundo semestre, el $42 \%$ (5/12), y el mayor diagnóstico viral se asoció con el pico de bronquiolitis en mayo (Ucrós, et al., 1997).

En otro estudio del 2001 en esta misma institución se encontró que el VSR tenía una prevalencia similar a la de otros países en menores de 24 meses de edad con síntomas sugestivos de enfermedad por VSR (Vásquez \& Reyes, 2001).

En el estudio de casos y controles prospectivo denominado "Factores de riesgo para infección respiratoria aguda baja grave en Bogota, 2001", realizado por el INS en colaboración con la Univesidad de La Sabana entre noviembre de 2000 y agosto de 2001 en una población de 638 niños entre los 2 meses y los 5 años que cumplían con los criterios de la OMS para la definición de IRA, se recolectó muestra nasofaríngea en 114 de ellos y 85,9 \% (98/114) de ellas resultaron positivas; en 41,8\% de estas se aisló el VSR. Entre los factores más importantes relacionados con la detección del virus se señaló "el compartir la cama" y la presencia de fumadores en la vivienda (Jaimes, et al., 2003).

En el Hospital Militar Central de Bogotá se llevó a cabo otro estudio en niños que consultaban con IRA, con el fin de determinar la frecuencia de los virus respiratorios causantes 
de la enfermedad. En este estudio transversal realizado entre marzo de 2000 y octubre de 2001. Se tomaron muestras de 139 niños, de las cuales 125 fueron aptas para el análisis virológico, y de estas $40 \%(50 / 125)$ resultó positivo para algún tipo de virus, siendo el VSR el más frecuente, con 30,4 \% (38 pacientes); además, se encontró que el VSR era el causante de $34,5 \%$ (20 casos) de las neumonías y que la infección se presentaba principalmente entre los meses de abril y junio (Herrera-Rodríguez, et al., 2007).

En el 2008, el Laboratorio de Salud Pública de la ciudad, conjuntamente con la Universidad de los Andes, desarrolló un protoloco de RT-PCR anidada para la detección y tipificación del VSR en 30 muestras de menores de 5 años; estas muestras se obtuvieron entre julio de 2005 y enero de 2006 y procedían de diferentes hospitales de Bogotá. El uso de la técnica estadarizada en el protocolo permitió establecer que de las 25 muestras positivas para VSR 16 correspondían a VSR tipo B, dos a VSR A y en siete se detectaron ambos subtipos del VSR (Mojica, et al., 2008).

El INS y el Ministerio de la Proteccion Social hicieron un estudio de los agentes asociados con casos de IRA a partir de los datos de la vigilancia clínica y de laboratorio en el país. En él se encontró que entre septiembre y diciembre del 2003 se reportaron 64 casos de IRA, de los cuales 21 (33\%) fueron fatales. De 25 pacientes cuyas muestras se analizaron, la mayoría tenía influenza y tres, VSR. El pico ocurrió con el cambio en la circulación de la cepa de la influenza predominante (Herrera, et al., 2008).

Un grupo de investigadores de la Universidad Javeriana hizo un estudio para determinar la presencia del VSR y otros virus respiratorios en menores de 15 años que acudieron durante el 2008 al servicio de urgencias pediátricas del Hospital San Ignacio. En los 451 pacientes evaluados, el VSR fue el virus causante de la mayor proporción $(65,8 \%)$ de IRA (297). El 93,3 \% de los pacientes era menor de 1 año y el pico de infección por VSR fue en abril ( $28 \%$ de 257 pacientes analizados) (Bastidas, 2009).

En otro estudio descriptivo restrospectivo de la Universidad Militar se describió el comportamiento clínico de pacientes pediátricos ingresados en la unidad de cuidados intensivos del Hospital Universitario Clínica San Rafael de Bogotá con infección por VSR durante el primer semestre de 2010 y 2011. Del total de 387 pacientes hospitalizados (182 en 2010 y 205 en 2011) en 91 se observó infeccion por VRS, es decir, $18 \%$ y $27 \%$ de todas las hospitalizaciones en la unidad de cuidados intensivos pediátricos durante 2010 y 2011, respectivamente (Castro, 2012).

El Laboratorio de Salud Pública de Bogotá propuso un modelo matemático epidemiológico con periodicidad forzada que fue comparado con los datos de la presencia del VSR en niños menores de 5 años en la ciudad entre 2005 y 2010. El modelo matemático explicó en forma razonable el comportamiento cíclico de los casos de niños infectados con el VSR, estableciéndose que la mayoría ocurría alrededor del mes de mayo, sin embargo, evidenció un comportamiento atípico del VSR en el 2007 (Aranda-Lozano, et al., 2013).
El trabajo denominado "Identificación de la presencia de dos paramixovirus humanos (VSR y metapneumovirus humano) en muestras de secreciones respiratorias recolectadas durante la vigilancia centinela en Colombia del 2000 al 2009", es un estudio de tipo descriptivo retrospectivo en el cual se analizó un grupo de muestras recolectadas en Bogotá en el marco del programa de vigilancia centinela de virus respiratorios en Colombia del INS entre el 2000 y el 2009. El estudio estimó una proporción de 45,7 \% de infección por VSR, con predomino del subtipo A, seguido por el subtipo B y $20,9 \%$ de coinfecciones con ambos subtipos. Además, los dos subtipos de VSR presentaron un comportamiento alternante en los años del estudio (Avila, 2014).

En un estudio descriptivo retrospectivo de la Universidad del Rosario para caracterizar la población con diagnóstico de bronquiolitis en la clínica infantil de Colsubsidio, se evaluaron 1.230 historias clínicas en el estudio, de las cuales 906 cumplían con los criterios de inclusión y en 36 \% (327) el diagnóstico se había establecido mediante panel viral; la edad promedio de los participantres fue de 5,5 meses y el principal agente aislado fue el VSR, en una proporción de $41 \%$ (Arraut \& Lesmes, 2014).

En otro estudio descriptivo de corte transversal de la Universidad Javeriana, se analizaron restrospectivamente las historias clínicas de menores de 2 años que asistieron con diagnóstico de IRA a la Unidad Pediátrica de los Andes entre 2011 y 2012 y a los que les solicitó examen de laboratorio para VSR o adenovirus. El porcentaje de muestras positivas para VSR fue de 42,6 \% (388/911). Se encontró, además, que en los meses de marzo, abril y mayo la frecuencia de casos de IRA por VSR era mayor y se hicieron evidentes pequeños picos durante los meses de septiembre a octubre $y$, en especial, en el mes de diciembre (Páez, 2013).

La Universidad Militar y la Universidad Nacional llevaron a cabo cinco estudios estrechamente relacionados en una misma cohorte de niños menores de tres años hospitalizados por IRA baja en la Fundación Hospital de La Misericordia, hospital universitario de atención terciaria ubicado en Bogotá (Gamba-Sánchez, et al., 2016; Rodríguez, et al., 2013; Rodríguez, et al., 2015; Rodríguez, et al., 2014; Rodríguez-Martínez, et al., 2015). Se hizo un muestreo por conveniencia entre todos los niños menores de 3 años hospitalizados, a quienes se les recolectaron aspirados nasofaríngeos para la detección de VSR y adenovirus durante un período de dos años entre mayo de 2009 y mayo de 2013. En las historias clínicas de los pacientes positivos para VSR o adenovirus se obtuvieron los datos correspondientes a la fecha de ingreso, la fecha de la toma del aspirado nasofaringeos, la edad, las comorbilidades, los requerimientos de oxígeno, el ingreso a la unidad de cuidados intensivos pediátrica y la mortalidad, entre otras; las variables predictoras incluidas en los estudios fueron edad, comorbilidades, mes del año en que se tomó la muestra de aspirados nasofaringeos y tipo de infección viral (VSR, adenovirus o infección mixta con VSR y 
adenovirus) (Gamba-Sánchez, et al., 2016; Rodríguez, et al., 2013; Rodríguez, et al., 2015; Rodríguez, et al., 2014; Rodríguez-Martínez, et al., 2015).

En el primer estudio de tipo analitico y retrospectivo que se hizo se analizaron 3.137 historias de niños hospitalizados por IRA baja, de las cuales $1.338(42,7 \%)$ eran de pacientes positivos para VSR y ADV. Durante los períodos de 2009 a 2010 y de 2010 a 2011 se detectaron $438(32,7 \%)$ y 900 $(67,3 \%)$ casos de IRA baja por VSR o adenovirus, respectivamente. Los pacientes positivos para VSR tenían una edad significativamente menor que aquellos negativos para VSR (8,0: 3,0-15,0 Vs. 12,0: 6,0-20,25, $\mathrm{p}<0,001)$. Por el contrario, los pacientes positivos para adenovirus tenían una edad significativamente mayor que aquellos con resultado negativo; 1.100 infecciones fueron causadas únicamente por el VSR, y de ellas 797 (72,5\%) se presentaron en marzo, abril y mayo, y 303 (27,5\%) en los otros meses (Rodríguez, et al., 2013).

En el segundo estudio se seleccionaron 2.147 historias de niños con resultado positivo para VSR y encontró una tasa de mortalidad por infección con VSR de 1,1\%; además, el aumento en las infecciones por VSR fue similar al estudio anterior, es decir, durante un periodo de tres meses (marzo a mayo). En el análisis multivariado se estableció el cáncer como comorbilidad y como factor predictor independiente de mortalidad en niños con infección por VSR; se determinaron, asimismo, otros factores de riesgo como la edad temprana, la prematuridad, las condiciones respiratorias y las cardiopatías congénitas preexistentes, factores que se asociaron con la gravedad de la infección por VSR en los niños (Rodríguez, et al., 2014).

Es el tercer estudio se correlacionó la actividad mensual del VSR con las variables meteorológicas (temperatura, humedad relativa, lluvia, velocidad del viento y radiación solar) mediante un análisis bivariado y un o multivariado. En el análisis bivariado se encontró una correlación entre el número de infecciones mensuales por VSR y las variables de temperatura y precipitación (Spearman: $p=0,730, p<0,01$ ) durante el periodo de tres meses (marzo a mayo), primera época de lluvias del año en Bogotá, y evidenció un aumento en los casos positivos de VSR durante el periodo de estudio (2009 a 2011). Las otras variables no tuvieron correlación alguna con las infecciones por VSR (Rodríguez, et al., 2015).

En el cuarto estudio se incluyeron 2.267 casos de niños con infección respiratoria aguda, de los cuales 1.991 (87,8\%) eran infecciones causadas únicamente por VSR, $205(9,0 \%)$, infecciones por adenovirus exclusivamente, y $71(3,1 \%)$, infecciones mixtas de VSR y adenovirus. Durante los meses de marzo a mayo se presentaron $1.266(63,6 \%)$ infecciones por VSR y el resto, $725(36,4 \%)$, ocurrieron en los restantes nueves meses del año. Con respecto a la gravedad de la enfermedad respiratoria, 488 (21,5\%) niños fueron ingresados en la unidad de cuidados intensivos pediátricos. Del total de 2.267 pacientes incluidos en el estudio, 2.110 (93,1\%) necesitaron administración de oxígeno suplementario, 106 (4,7\%), intubación endotraqueal, 596 (26,3\%), antibióticos, y 1.008 (44,5\%), oxígeno domiciliaria; a tasa mortalidad observada fue de $1,3 \%$ durante el periodo de estudio (Rodríguez-Martínez, et al., 2015).

Por último, el quinto trabajo consistió en un estudio analítico transversal durante un período de cinco años (2009 a 2013), en el cual seb evaluaron la asociación entre los parámetros meteorológicos y la actividad del VSR en esa población de niños hospitalizados en el Hospital de la Misericordia. Se analizaron 13.488 muestras, de las cuales 4.559 (33,8 \%) fueron positivas para VSR. Se encontró que la circulación del VSR fue continua a lo largo del período de cinco años, alcanzando un pico cada año durante un período de tres meses, entre marzo y mayo, el cual corresponde a la época de lluvias en la ciudad; también se evidencipo una relación directa entre el aumento de la temperatura y la lluvia y el número de casos de VSR. Las variables de humedad relativa, radiación y velocidad del viento no presentaron una correlación estadísticamente significativa con el número de casos positivos de VSR (Gamba-Sánchez, et al., 2016)2016.

En el Hospital Universitario Clínica San Rafael se realizó un estudio prospectivo de cohorte entre enero del 2014 y enero del 2015, con el fin de determinar factores predictores de la gravedad en menores de 5 años hospitalizados con diagnóstico de IRA baja a quienes se les tomaron muestras de aspirado nasofaríngeo para detectar virus respiratorios mediante la técnica de inmunofluorescencia indirecta. Se analizaron 1.180 pacientes con diagnóstico de IRA baja, de los cuales $416(35,3 \%)$ se seleccionaron por ser positivos para algún tipo de virus respiratorio. De estos 416 pacientes, $352(84,6 \%)$ tenían infecciones únicamente por VSR, 39 $(9,4 \%)$, infecciones exclusivamente con parainfluenza, $12(2,9 \%)$, infecciones solo con adenovirus, siete $(1,7)$ eran infecciones únicamente por influenza, y los seis restantes $(1,4 \%)$ tenían infecciones respiratorias mixtas; estas infecciones respiratorias virales, especialmente las producidas por VSR, con $74,1 \%$, ocurrieron durante un período de trexs meses entre marzo y mayo, época de lluvia en el país. Con respecto a la gravedad de la enfermedad respiratoria, se encontró que $86(20,7 \%)$ pacientes fueron ingresados en la unidad de cuidados intensivos pediátricos, y $67(16,1 \%)$ necesitaron intubación endotraqueal. Ninguno de los pacientes incluidos falleció debido a la infección respiratoria. De los 416 pacientes incluidos en el estudio, $165(39,7 \%)$ tuvieron una hospitalización media de más de siete días, $163(39,2 \%)$ recibieron antibióticos y $24(5,8 \%)$ requerieron soporte inotrópico o vasopresor. Además, se demostró que los antecedentes de sibilancias recurrentes, de hipertensión pulmonar y desnutrición como comorbilidad eran predictores de infecciones respiratorias agudas virales (Pedraza-Bernal, et al., 2016)

La Secretaría de Salud de Bogotá hizo uno de los pocos estudios encontrados en adultos con infecciones respiratorias agudas graves, en el cual se encontró, que como era de esperarse, el VSR no fue el principal agente en esta poblacion (Remolina, et al., 2015). 
En otro estudio en el Hospital Militar de Bogotá se buscó caracterizar a los pacientes de alto riesgo (prematuros menores de 35 semanas con enfermedad pulmonar crónica y aquellos con cardiopatías congénitas hemodinámicamente significativas) hospitalizados con infección por VSR en dicho hospital luego de haber recibido profilaxis con palivizumab o no. En este estudio observacional descriptivo prospectivo se analizaron 240 pacientes de los cuales el 44,58 \% fue positivo para el VSR (Acuña, et al., 2015). En el 2013 la prevalencia de hospitalización durante el año fue mayor en los meses de marzo a junio, con un pequeño pico al final del año.

La Universidad El Bosque de Bogotá llevó a cabo un estudio para predecir las semanas de inicio de los brotes de infección por el VSR en la ciudad entre 2005 y 2010 con base en variables climáticas (humedad, temperarura, sensación térmica, entre otras), las cuales se seleccionaron empleando el método bayesiano ingenuo. Los resultados de este trabajo muestran la relación de la humedad con el inicio del brote de VSR en Bogotá, que se produjo principalmente en el primer semestre de los años de estudio (GonzálezParra, et al., 2016).

\section{Estudios en Tunja, Bucaramanga y Cartagena}

En Bucaramanga se realizó un estudio para estimar la incidencia del VSR asociado a IRA en una población pediátrica. El estudio se hizo en el marco del programa de vigilancia centinela (Sivigila) de circulación de virus respiratorios en Colombia, e incluyó a pacientes atendidos en el servicio de urgencias del Hospital Universitario Ramón González Valencia con cuadro clínico compatible con IRA. Se recolectaron 70 muestras en las cuales se identificó un agente viral en $21,42 \%\left(\mathrm{IC}_{95 \%}: 12,9-33,2\right)$; el VSR se detectó en $10 \%\left(\mathrm{IC}_{95 \%}: 4,5-20,1\right)$ (Sarmiento, 2007) de la población estudiada durante dos años (2004 y 2005). El número de casos (7 casos) hace difícil hablar de periodicidad, pero al parecer se presentó en enero (la mayoría de los casos).

En Tunja se hio un estudio descriptivo para la caracterización epidemiológica de la IRAG (código 345 del Sivigila) entre julio de 2012 y julio de 2013. Se incluyeron pacientes de cualquier edad con infección respiratoria aguda grave internados en el Hospital San Rafael y en la Clínica Saludcoop de Tunja, a quienes se había tomado muestra de hisopado o aspirado nasofaríngeo. Se utilizaron los datos del Sivigila correspondientes a 530 muestras de hisopado nasofaríngeo procesadas mediante inmunofluorescencia directa. Se identificaron virus en 6,8 \% de los casos, $75 \%$ de los cuales fueron VSR (García, 2014). Los picos de detección fueron en mayo y junio.

En el estudio retrospectivo en Cartagena se evaluó la frecuencia de infección por VRS a partir de la revisión de 170 historias clínicas de pacientes pediátricos con sintomatología respiratoria y "sospecha de infección viral", la mayoría de ellos $(95,9 \%)$ hospitalizados. El VSR fue el virus más frecuentemente encontrado, con 40,53 \% de los casos (Díaz-Martínez \& Gaviria-Díaz, 2014).
En la Tabla 1 se resume la prevalencia del VSR en población pediátrica en Colombia estimada con base en los principales estudios revisados, con énfasis en los datos reportados en menores de 5 años, principal población en riesgo de enfermedad grave por VSR. No se incluyeron los estudios con información duplicada sobre la prevalencia de VSR.

En la tabla 2 se presentan los estudios que han evaluado la periodicidad durante periodos de más de un año de la infección por VSR en diferentes ciudades de Colombia.

\section{Discusión y conclusiones}

Un hallazgo común en la mayoría de estudios es que el VSR fue el virus más frecuentemente detectado en IRA baja en menores de 5 años, seguido por el de influenza A y adenovirus (Tabla 1). Las coinfecciones de VSR y otros virus respiratorios reportadas en algunos de los estudios fueron menos de $10 \%$. Sin embargo, resulta llamativa la alta frecuencia de la coinfección con Streptococcus aureus y con C. trachomatis, el principal diagnóstico diferencial del VSR en la neumonías en menores de seis meses, la cual se estimó en un solo estudio (Núñez, et al., 1988). Por otra parte, las prevalencias más bajas de VSR, diferentes del resto del país (7-13 \%), se registraron en Tunja, Bucaramanga y Cali. En Tunja y Bucaramanga resulta difícil corroborar este resultado, pues solo se encontró un estudio por ciudad. Las diferencias en el diseño y el propósito de los estudios o los métodos de confirmación del VSR de las investigaciones en Cali podrían explicar las diferencias en la frecuencia del VSR con respecto a otros estudios en Colombia. Dos de las investigaciones en Cali correspondieron a seguimientos prospectivos de cohortes con vigilancia activa de los episodios de IRA en niños sanos (Borrero, et al., 1990; Nolan, et al., 2015), en tanto que el resto de estudios en Colombia han sido de corte transveral en población de menores hospitalizados. Aunque los otros dos estudios de Cali con prevalencias de VSR de 4 y $9 \%$, también se hicieron en pacientes de IRA baja hospitalizados, la confirmación de la infección por VSR se hizo mediante la detección de anticuerpos fijadores del complemento (Berman, et al., 1983), comparada con la detección por IFI o RT-PCR utilizada en los demás estudios colombianos. Por el contrario, cuando se compararon estudios similares de vigilancia pasiva de IRA en menores hospitalizados y la detección por inmunofluorescencia, la prevalencia en Cali (Piñeros, et al., 2013) fue más alta (30\%) y comparable a la reportada en Bogotá (30 - $52 \%$ ), Medellín (42\%), Manizales (25\%) y Cartagena (41\%) o a la estimación con base en todos los estudios (22\%).

Con respecto a la periodicidad de la trasmisión del VSR en Colombia, los resultados consignados en la Tabla 2 mostraron un aumento en la frecuencia de los casos por VSR a partir del mes de marzo, con picos durante los meses de abril a junio, y una disminución posterior durante los meses de agosto a septiembre. Esta situación ocurre principalmente en ciudades como Bogotá, Tunja y Manizales (Barbosa- 
Tabla 1. Prevalencia del VSR en Colombia en población pediátrica con IRA

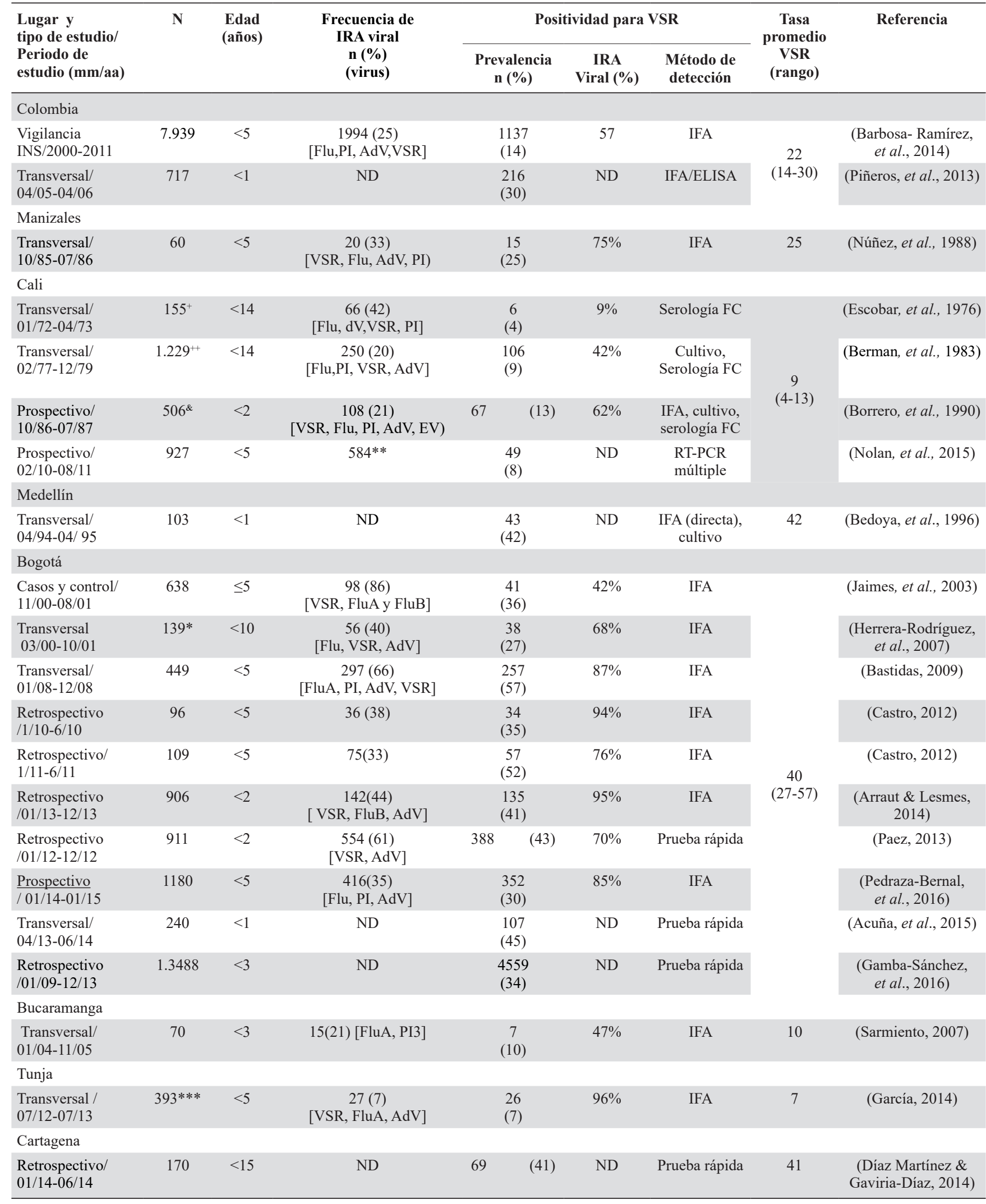

$* 85 \%$ era menor de tres años (118/139); ${ }^{* *}$ Episodios de IRA baja; $* * * 60 \%$ era menor de un año $(234 / 393) .{ }^{+} 91 \%$ era menores de 5 años $(141 / 155)++$ 66 \% era menor de 2 años (811/1229); ' Episodios de IRA baja en 304 niños; ND: no determinado o sin dato; IFA: ensayo de Inmunofluorescencia; Flu: virus de la Influenza; FluA: virus de la Influenza tipo A; FluB: virus de la Influenza tipo B; AdV: adenovirus; PI: parainfluenza; PI3: parainfluenza tipo 3; EV: enterovirus 
Tabla 2. Principales estudios que han evaluado la periodicidad del VSR en Colombia

\begin{tabular}{|c|c|c|c|c|c|c|c|}
\hline Lugar (ciudad) & Tipo de estudio & $\begin{array}{l}\text { Edad } \\
\text { (años) }\end{array}$ & $\begin{array}{c}\text { + VSR / } \\
\text { Total analizados } \\
\text { (\%) }\end{array}$ & $\begin{array}{l}\text { Periodo de } \\
\text { estudio }\end{array}$ & Método & Frecuencia (Picos) & Referencia \\
\hline Nacional & $\begin{array}{l}\text { Descriptivo - } \\
\text { retrospectivo }\end{array}$ & $0->65$ & $\begin{array}{c}1358 / 2799 \\
(48,5)\end{array}$ & 2000 a 2011 & IFA & $\begin{array}{c}\text { Abril a junio } \\
\text { Septiembre a } \\
\text { Noviembre }\end{array}$ & $\begin{array}{l}\text { (Barbosa Ramirez, } \\
\text { et al., 2014) }\end{array}$ \\
\hline $\begin{array}{l}\text { Bogotá D,C. } \\
\text { Barranquilla, Atlántico } \\
\text { Medellín, (Antioquia) } \\
\text { Bucaramanga (Santander) } \\
\text { Pereira (Armenia) }\end{array}$ & Descriptivo & $<1$ & $\begin{array}{c}216 / 717 \\
(30,0)\end{array}$ & $\begin{array}{c}\text { Abr, } 2005 \text { a } \\
\text { Abr, } 2006\end{array}$ & $\begin{array}{l}\text { - ELISA } \\
\text { - IFA }\end{array}$ & $\begin{array}{c}\text { Abril a junio } \\
\text { (Bogotá, Medellín) } \\
\text { Todo el año } \\
\text { (Cali, Barranquilla, } \\
\text { Bucaramanga) }\end{array}$ & $\begin{array}{l}\text { (Piñeros, } \\
\text { et al., 2013) }\end{array}$ \\
\hline \multirow[t]{9}{*}{ Bogotá D,C. } & $\begin{array}{l}\text { Descriptivo, } \\
\text { longitudinal y } \\
\text { prospectivo }\end{array}$ & $<16$ & $\begin{array}{l}32 / 46 \\
(69,5)\end{array}$ & 1990 a 1994 & ELISA & Abril a junio & (Ucrós, et al., 1997) \\
\hline & Transversal & $<2$ & $\begin{array}{c}38 / 139 \\
(27,3)\end{array}$ & $\begin{array}{l}\text { Mar } 2000 \text { a } \\
\text { Oct } 2001\end{array}$ & $\begin{array}{l}\text { Cultivo / } \\
\text { IFA }\end{array}$ & Abril a junio & $\begin{array}{l}\text { (Herrera-Rodríguez, } \\
\text { et al., 2007) }\end{array}$ \\
\hline & $\begin{array}{c}\text { Observacional } \\
\text { retrospectivo de } \\
\text { cohorte transversal }\end{array}$ & $<15$ & $\begin{array}{c}257 / 451 \\
(57,0)\end{array}$ & 2008 & IFA & Marzo a mayo & (Bastidas, 2009) \\
\hline & $\begin{array}{l}\text { Modelo } \\
\text { matematico }\end{array}$ & $<5$ & ------ & $\begin{array}{l}\text { Mar } 2005 \\
\text { a } 2010\end{array}$ & IFI & Mayo & $\begin{array}{l}\text { (Aranda-Lozano, } \\
\text { et al., 2013) }\end{array}$ \\
\hline & $\begin{array}{l}\text { Descriptivo de } \\
\text { cohorte transversal }\end{array}$ & $<2$ & $\begin{array}{l}388 / 911 \\
(42,7)\end{array}$ & 2012 & $\begin{array}{l}\text { Prueba } \\
\text { Rapida }\end{array}$ & $\begin{array}{c}\text { Marzo a mayo } \\
\text { Septiembre a } \\
\text { diciembre }\end{array}$ & (Paez, 2013) \\
\hline & Descriptivo & $>18$ & $\begin{array}{l}5 / 91 \\
(5,4)\end{array}$ & 2012 & Microrray & Abril a mayo & $\begin{array}{l}\text { (Remolina, } \\
\text { et al., 2015) }\end{array}$ \\
\hline & Prospectivo & $<5$ & $\begin{array}{c}352 / 416 \\
(84,6)\end{array}$ & $\begin{array}{l}\text { Ene } 2014 \text { a } \\
\text { Ene } 2015\end{array}$ & IFA & Marzo a mayo & $\begin{array}{l}\text { (Pedraza-Bernal, } \\
\text { et al., 2016) }\end{array}$ \\
\hline & $\begin{array}{c}\text { Observacional, } \\
\text { descriptivo } \\
\text { Prospectivo }\end{array}$ & $<1$ & $\begin{array}{c}107 / 240 \\
(44,6)\end{array}$ & $\begin{array}{l}\text { Abr, } 2013 \text { a } \\
\text { Jun, } 2014\end{array}$ & $\begin{array}{l}\text { Prueba } \\
\text { Rapida }\end{array}$ & Marzo a junio & $\begin{array}{l}\text { (Acuña, } \\
\text { et al., 2015) }\end{array}$ \\
\hline & Analítico & $<3$ & $\begin{array}{l}4559 / 13488 \\
(33,8)\end{array}$ & 2009 a 2013 & $\begin{array}{l}\text { Prueba } \\
\text { Rapida }\end{array}$ & Marzo a mayo & $\begin{array}{l}\text { (Gamba-Sanchez, } \\
\text { et al., 2016) }\end{array}$ \\
\hline Medellin, Antioquia & Descriptivo & $<1$ & $\begin{array}{l}43 / 103 \\
(41,7)\end{array}$ & $\begin{array}{l}\text { Abr } 1994 \\
\text { a } 1995\end{array}$ & $\begin{array}{l}\text { IFA, } \\
\text { Cultivo }\end{array}$ & Nov a Ene & $\begin{array}{l}\text { (Bedoya, } \\
\text { et al., 1996) }\end{array}$ \\
\hline Cali, Valle & Transversal & $<2$ & $\begin{array}{c}106 / 1229 \\
(9,0)\end{array}$ & $\begin{array}{l}\text { Feb } 1977 \text { a } \\
\text { Feb } 1979\end{array}$ & $\begin{array}{l}\text { Cultivo, } \\
\text { Serologia, } \\
\text { FC }\end{array}$ & Dic a Feb & $\begin{array}{l}\text { (Berman, } \\
\text { et al., 1983) }\end{array}$ \\
\hline Tunja, Boyacá & $\begin{array}{l}\text { Descriptivo } \\
\text { retrospectivo }\end{array}$ & $<5$ & $\begin{array}{c}27 / 530 \\
(75,0)\end{array}$ & $\begin{array}{l}\text { Jul } 2012 \text { a } \\
\text { Jul } 2013\end{array}$ & IFA & Mar a Jul & (García, 2014) \\
\hline Bucaramanga, Santander & Transversal & $<3$ & $\begin{array}{l}7 / 70 \\
(10,0)\end{array}$ & $\begin{array}{l}\text { Ene, } 2004 \text { a } \\
\text { Nov, } 2005\end{array}$ & IFA & $\begin{array}{l}\text { Ene a Feb } \\
\text { May a Ago }\end{array}$ & (Sarmiento, 2007) \\
\hline Manizales, Caldas & Transversal & $\leq 1-60$ & $\begin{array}{l}15 / 60 \\
(25,0)\end{array}$ & $\begin{array}{l}\text { Oct, } 1985 \text { a } \\
\text { Jul, } 1986\end{array}$ & IFA & Ene a Jul & (Núñez, et al., 1988) \\
\hline
\end{tabular}

En esta tabla se incluyen los estudios que han evaluado la prevalencia de VSR por lo menos durante un año y que no tienen informacion redundante. ------Sin dato; IFA: ensayo de inmunofluorescencia

Ramírez, et al., 2014; Gamba-Sánchez, et al., 2016; García, 2014; Núñez, et al., 1988). En otras ciudades del país como Cali, Medellín y, quizás, Bucaramanga la frecuencia de los picos de presencia del VSR se concentró principalmente durante los meses de diciembre a febrero (Bedoya, et al., 1996; Berman, et al., 1983; Sarmiento, 2007), lo cual indica que la periodicidad del VSR en Colombia se da a lo largo del año, con picos que varían ligeramente según la ciudad.
Aunque no es completamente clara la asociación entre la frecuencia de los casos por VSR y los factores meterológicos como la temperatura, la humedad o la lluvia, las temporadas de lluvias y las temperaturas cálidas favorecen la replicación, la estabilidad y la transmisión del virus todo el año (Rodríguez, et al., 2015). En los estudios realizados en el país tanto de cohorte como descriptivos el aumento en la frecuencia de casos por VSR concidió con el inicio de las 
lluvias en la región andina, en la cual la primera temporada de lluvias ocurre entre marzo y mayo y la segunda entre septiembre y noviembre (Barbosa-Ramírez, et al., 2014; Gamba-Sánchez, et al., 2016).

La presente revisión permite concluir que hay un importante número de trabajos que han evaluado la prevalencia del VSR en Colombia y que pueden ser útiles para estimar la carga de la enfermedad por este patógeno en el país. Los estudios gubernamentales parecen ser la mejor fuente para estimar la prevalencia de VSR en la población pediátrica con IRA baja en Colombia. Entre los otros estudios, resalta el único estudio prospectivo que logramos detectar, en el cual se estimó la incidencia de la enfermedad por VSR (Nolan, et al., 2015). En futuros estudios que pretendan estimar la carga de la enfermedad y la prevalencia del VSR en Colombia, se deberá tener en cuenta la heterogeneidad y la periodicidad de la presentación del VSR en las diferentes regiones del país. Asimismo, es importante hacer estudios para determinar la carga de la enfermedad en el país que tengan en cuenta los estudios previos sobre el palivizumab (Rueda, et al., 2013) y los efectos a largo plazo del VSR, tales como el incremento de asma (Lambert, et al., 2014).

Se desconocen aún muchos aspectos (poblacionales, estacionales, efectos del medio ambiente, factores de riesgo, etc.), sobre el VSR, necesarios para mejorar el manejo de la enfermedad producida por este virus. La información sobre cuándo inicia y termina la temporada del VSR en el país o la región, así como sobre la carga de la enfermedad en la población general y en especial en los grupos de riesgo (menores de un año), permitiría optimizar el tratamiento y las medidas profilácticas (vacuna o inmunoprofilaxis) contra el VSR. Teniendo en cuenta las limitaciones del país en cuanto a la calidad de la información epidemiológica y virológica sobre el VSR, las autoridades de salud son conscientes de la necesidad de asumir el reto de mejorarla introduciendo cambios en la vigilancia, la representatividad de la muestra, la consolidación y la generación de fuentes de información y deconsultadas (Barbosa, et al., 2014). Debe enfatizarse la necesidad de conocer más a fondo la epidemiología de las infecciones respiratorias agudas en nuestra población, incluidos datos precisos sobre su incidencia, a través de estudios multicéntricos con definiciones de caso homogéneas que permitan entender mejor la prevalencia del VSR en Colombia.

\section{Agradecimientos}

Nos gustaría agradecer a los participantes del seminario taller sobre "Integración intersectorial en la investigación para la salud" organizado por el CIDEIM con el apoyo del Programa Especial TDR de la OMS, el Banco Mundial, la UNDO y la UNICEF), y en particular a las personas que trabajaron en el tema: "Colombia en la preparación para la introducción de la vacuna contra el Virus Sincitial Respiratorio", por sus aportes y enriquecedoras discusiones.

\section{Contribución de los autores}

JB realizó la revisión de los trabajos del Instituto Nacional de Salud y de los artículos de estudios provenientes de Bogotá. BP hizo la revisión primaria de los artículos de otras regiones del pais. LA y FIQ se encargaron del análisis preliminar de un subgrupo de las referencias. EL realizó el análisis general de todas las referencias. MAF hizo la búsqueda de la mayoría de los artículos sujetos a revisión y estructuró el cuerpo del artículo. Todos los autores revisaron la versión final del manuscrito y aprobaron su contenido.

\section{Conflicto de intereses y financiación}

Declaramos no tener ningún conflicto de intereses. Este trabajo fue financiado parcialmente por la Pontificia Universidad Javeriana de Bogotá, y por el Programa Especial TDR de la OMS, el Banco Mundial, la UNDO y la UNICEF (contrato 2015/583947-0).

\section{Referencias}

Acuña, R.H., Colmenares, A., Arismiendi, M. 2015. Impacto de la profilaxis con Palivizumab en la hospitalizacion de recién nacidos de alto riesgo para infección por virus sincitial respiratorio en el Hospital Militar Central, Pediatría. Universidad Militar Nueva Granada. Fecha de consulta: 30 de septiembre de 2016. Disponible en: http://repository. unimilitar.edu.co/handle/10654/6222.

Aranda-Lozano, D.F., González-Parra, G.C., Querales, J. 2013. Modelling respiratory syncytial virus (RSV) transmission children aged less than five years-old. Rev Salud Pública (Bogota). 15: 689-700.

Arango, A.E., Jaramillo, S., Pérez, J., Ampuero, J.S., Espinal, D., Donado, J., Felices, V., García, J., Laguna-Torres, A. 2015. Influenza-like illness sentinel surveillance in one hospital in Medellín, Colombia. 2007-2012. Influenza and other respiratory viruses. 9: 1-13.

Arraut, P.B., Lesmes, A.C. 2014. Caracterización de la población con bronquiolitis en la Clinica Infantil Colsubsidio en el año 2013, Pediatría. Universidad del Rosario, Fecha de consulta: 30 de septiembre de 2016. Disponible en: http://repository.urosario.edu.co/bitstream/handle/10336/ 10515/53106146-2015.pdf?sequence $=1$.

Ávila, L.V.2014. Identificación de la presencia de dos paramixovirus humanos (virus sincitial respiratorio humano y metanuemovirus humano) en muestras de secreciones respiratorias recolectadas durante la vigilancia centinela en Colombia del 2000 al 2009. Universidad Nacional de Colombia. Fecha de consulta: 30 de septiembre de 2016. Disponible en: http:// www.bdigital.unal.edu.co/46137/1/1032374854.2014.pdf

Barbosa, J., Daza, J., Malo, D. 2014. Vigilancia epidemiológica y virológica de influenza y otros virus respiratorios en Colombia. 2000 - 2014. Instituto Nacional de Salud, Bogotá, Colombia.

Barbosa-Ramírez, J., Pulido-Domínguez, P., Rey-Benito, G., Méndez-Rico, J., Castellanos, J., Páez-Martínez, A. 2014. Human respiratory syncytial virus and metapneumovirus in patients with acute respiratory infection in Colombia, 2000 - 2011. Rev Panam Salud Pública. 36: 101-109.

Bardach, A., Rey-Ares, L., Cafferata, M.L., Cormick, G., Romano, M., Ruvinsky, S., Savy, V. 2014. Systematic review and metaanalysis of respiratory syncytial virus infection epidemiology in Latin America. Rev Med Virol. 24: 76-89. 
Bastidas, M.L. 2009. Frecuencia de virus causantes de infección respiratoria aguda baja en pacientes pediátricos del Hospital Universitario San Ignacio durante el año 2008, Microbiología. Pontificia Universidad Javeriana. Fecha de consulta: 30 de septiembre de 2016. Disponible en: http:// www.javeriana.edu.co/biblos/tesis/ciencias/tesis335.pdf

Bedoya, V.I., Abad, V., Trujillo, H. 1996. Frequency of respiratory syncytial virus in hospitalized infants with lower acute respiratory tract infection in Colombia. Pediatric Infectious Disease Journal. 15: 1123-1124.

Berman, S., Dueñas, A., Bedoya, A. 1983. Acute lower respiratory tract illnesses in Cali, Colombia: A two-year ambulatory study. Pediatrics. 71: 210-218.

Borrero, I.H., Fajardo, L.P., Bedoya, A.M., Zea, A., Carmona, F., De Borrero, M.F. 1990. Acute respiratory tract infections among a birth cohort of children from Cali, Colombia, who were studied through 17 months of age. Reviews of Infectious Diseases. 12: S950-S956.

Castro, J.P. 2012. Caracterización del virus sincitial respiratorio en la unidad de cuidados intensivos pediátricos del Hospital Universitario Clínica San Rafael de Bogotá en el periodo de enero 1 a junio 30 de los años 2010 y 2011. Pediatría. Universidad Militar Nueva Granada. Fecha de consulta: 30 de septiembre de 2016. Disponible en: http:// repository.unimilitar.edu.co/bitstream/10654/10041/2/ CastroEspitiaJhohanPaul2012.pdf

Chu, H.Y., Steinhoff, M.C., Magaret, A., Zaman, K., Roy, E., Langdon, G., Formica, M.A., Walsh, E.E., Englund, J.A. 2014. Respiratory Syncytial Virus Transplacental Antibody Transfer and Kinetics in Mother-Infant Pairs in Bangladesh. Journal of Infectious Diseases. 210: 1582-1589.

Díaz-Martínez, I., Gaviria-Díaz, A. 2014. Frequencia de infeccion por virus sincitial respiratorio y virus influenza y sur relación con características clínico-radiológicas y sociodemográficas asociadas. Facultad de Ciencias de la Salud, Programa de Bacteriología. Universidad San Buenaventura. Fecha de consulta: 30 de septiembre de 2016. Disponible en: http:// bibliotecadigital.usbcali.edu.co/jspui/handle/10819/2578

Escobar, J.A., Dover, A.S., Dueñas, A., Leal, E., Medina, P., Argüello, A., de Gaiter, M., Greer, D.L., Spillman, R., Reyes, M.A. 1976. Etiology of respiratory tract infections in children in Cali, Colombia. Pediatrics. 57: 123-130.

Gamba-Sánchez, N., Rodríguez-Martínez, C.E., Sossa-Briceño, M.P. 2016. Epidemic activity of respiratory syncytial virus is related to temperature and rainfall in equatorial tropical countries. Epidemiol Infect. 144 (10): 2057-63.

García, C.M. 2014. Caracterización epidemiológica de la infección respiratoria aguda grave y circulación viral en Boyacá, julio de 2012 a julio de 2013, Departamento Salud Pública, Facultad de Medicina. Universidad Nacional de Colombia. Fecha de consulñta: 30 de septiembtre de 2016. Disponible en: http://www.bdigital.unal.edu.co/43123/

Giersing, B., Modjarad, K., Kaslow, D., Moorthy, V. 2016. Report from the World Health Organization's Product Development for Vaccines Advisory Committee (PDVAC) meeting, Geneva, 7-9th Sep 2015. Vaccine. 34: 2865-2869.

González-Parra, G., Querales, J.F., Aranda, D. 2016. Predicción de la epidemia del virus respiratorio sincitial en Bogotá D.C. utilizando variables climatológicas. Biomédica. 36 (3): $378-389$
Graham, B.S. 2016. Vaccines against respiratory syncytial virus: The time has finally come. Vaccine. 34: 3535-3541.

Herrera, D., de la Hoz, F., Velandia, M. 2008. Severe respiratory disease and its relationship with respiratory viruses in Colombia. International Journal of Infectious Diseases. 12: 139-142.

Herrera-Rodríguez, D.H., De La Hoz, F., Marino, C., Ramírez, E. 2007. Respiratory virus in children aged less than 10 years old suffering from respiratory infection in the Hospital Militar Central in Bogotá from 2000-2001. Rev Salud Pública (Bogota). 9: 576-586.

Higgins, D., Trujillo, C., Keech, C. 2016. Advances in RSV vaccine research and development - A global agenda. Vaccine. 34 (26): 2870-2875.

Jaimes, M.B., Cáceres, D.C., de la Hoz , F., Gutiérrez, C., Herrera, D., Pinilla, J., Porras, A., Rodríguez, F., Velandia, M. 2003. Factores de riesgo para infección respiratoria aguda baja grave en Bogotá, 2001. Biomédica. 23: 283-292.

Lambert, L., Sagfors, A.M., Openshaw, P.J., Culley, F.J. 2014. Immunity to RSV in Early-Life. Front Immunol. 5: 466.

Ministerios de Salud. 2014. Actualización de la recomendación sobre el uso de Palivizumab, 2013-2014. Fecha de consulta: 30 de septiembre de 2016. Disponible en: http:// fundacioncanguro.co/files/Actualizacin_2013_2014_ GPC_Premat.pdf

Modjarrad, K., Giersing, B., Kaslow, D.C., Smith, P.G., Moorthy, V.S. 2015. WHO consultation on Respiratory Syncytial Virus Vaccine Development Report from a World Health Organization Meeting held on 23-24 March 2015. Vaccine. 34: 190-197.

Mojica, M.F., Escobar, M.F., Escalante, M.P., Jaramillo, C.A., Delgado, M. 2008. Detección y tipificación del virus sincitial respiratorio mediante la técnica RT- PCR anidada en pacientes con infección respiratoria aguda. Revista Instituto Nacional Enfermedades Respiratorias de México. 21: 92-98.

Nolan, T., Borja-Tabora, C., López, P., Weckx, L., UlloaGutiérrez, R., Lazcano-Ponce, E., Kerdpanich, A., Weber, M.A., Mascarenas de Los Santos, A., Tinoco, J.C., Safadi, M.A., Seng, L.F., Hernández-de Mezerville, M., Faingezicht, I., Cruz-Valdéz, A., Feng, Y., Li, P., Durviaux, S., Haars, G., Roy-Ghanta, S., Vaughn, D.W., Taylor, S. 2015. Prevalence and Incidence of Respiratory Syncytial Virus and Other Respiratory Viral Infections in Children Aged 6 Months to 10 Years With Influenza-like Illness Enrolled in a Randomized Trial. Clin Infect Dis. 60: e80-89.

Núñez, R.M., Dugue, J., Henríquez, C.W., Villegas, A.L., Niño, J.U., De Gonzáles, B. 1988. Viral and chlamydial etiology of acute infections of the lower respiratory tract in Colombian children. The Pediatric infectious disease journal. 7: 69-70.

Páez, L.D. 2013. Prevalencia de virus sincitial respiratorio y adenovirus en un grupo de niños menores de dos años de la Unidad Pediátrica De Los Andes, Microbiologíaa. Pontificia Universidad Javeriana. Fecha de consulta: 30 de septiembre de 2016.Disponible en: https://repository.javeriana.edu.co/ bitstream/handle/10554/13293/PaezCardozoLeiderDorey 2013.pdf? sequence $=1 \&$ isAllowed $=y$ 
Pedraza-Bernal, A.M., Rodríguez-Martínez, C.E., AcuñaCordero, R. 2016. Predictors of severe disease in a hospitalized population of children with acute viral lower respiratory tract infections. Journal of Medical Virology. 88: 754-759.

Piñeros, J.G., Baquero, H., Bastidas, J., García, J., Ovalle, O., Patiño, C.M., Restrepo, J.C. 2013. Respiratory syncytial virus infection as a cause of hospitalization in population under 1 year in Colombia. J Pediatr (Rio J). 89: 544-548.

Remolina, Y.A., Ulloa, M.M., Vargas, H., Díaz, L., Gómez, S.L., Saavedra, A., Sánchez, E., Cortés, J.A. 2015. Viral Infection in Adults with Severe Acute Respiratory Infection in Colombia. PLoS One. 10: e0143152.

Rodríguez, C., Rodríguez, D.A., Cárdenas, A., Quilaguy, I.E., Mayorga, L.Y., Falla, L.M., Sossa, M. 2013. Infección por virus sincitial respiratorio y por adenovirus en una poblacion de niños colombianos menores de 3 años hospitalizados por infección respiratoria aguda baja. Revista Médica Sanitas. 16: $16-24$.

Rodriguez-Martinez C.E., Sossa-Briceño M.P., Acuña-Cordero R. 2015. Relationship between meteorological conditions and respiratory syncytial virus in a tropical country. Epidemiol Infect. 143 (12): 2679-86.

Rodríguez, D.A., Rodríguez-Martínez, C.E., Cárdenas, A.C., Quilaguy, I.E., Mayorga, L.Y., Falla, L.M., Niño, G. 2014. Predictors of severity and mortality in children hospitalized with respiratory syncytial virus infection in a tropical region. Pediatr Pulmonol. 49: 269-276.
Rodríguez-Martínez, C.E., Rodríguez, D.A., Nino, G. 2015. Respiratory syncytial virus, adenoviruses, and mixed acute lower respiratory infections in children in a developing country. J Med Virol. 87: 774-781.

Rueda, J.D., Rosselli, D., Ruiz-Peláez, J.G. 2013. CostEffectiveness of Respiratory Syncytial Virus Infection (RSV) Prophylaxis with Palivizumab in Preterm Infants in Colombia. Coyuntura Económica: Investigación Económica y Social. 43: 137-151.

Sarmiento, I.C. 2007. Estimación de la Incidencia del VSR asociado a infección respiratoria aguda en una población pediátrica del Hospital Universitario Ramón González Valencia, Departamento de Pediatría, Facultad de Medicina. Universidad Industrial de Santander. Fecha de consulta: 30 de septiembre de 2016. Disponible en: http://repositorio. uis.edu.co/jspui/bitstream/123456789/10202/2/124014.pdf

Ucrós, S., Dueñas, E., Gutiérrez, M. 1997. Calendario y variación estacional de las afecciones respiratorias en Santa Fe de Bogotá. Revista Colombiana de Neumología. 9: 124-132.

Vásquez, C., Reyes, M. 2001. Prevalencia de virus sincitial respiratorio (VSR) en pacientes de alto riesgo, menores de 24 meses de edad con síntomas sugestivos de enfermedad por VSR y evaluación de costos. Actualizaciones Pediátricas. 11: 3-11.

Weber, M.W., Mulholland, E.K., Greenwood, B.M. 1998. Respiratory syncytial virus infection in tropical and developing countries. Trop Med Int Health. 3: 268-280. 